\title{
Response of the Saladette Tomato of Growth Determinate to Different Sources and Doses of Calcium in Greenhouse
}

\author{
Heidi Melania Medina Montenegro, Vicente Álvarez Mares, Leopoldo Partida Ruvalcaba*, \\ Jesús Antonio Martínez Sarabia, Jesús Adán León Terán \\ Universidad Tecnológica de Culiacán, carretera Culiacán-Imala km 2, en la ciudad educadora del saber, \\ Culiacán de Rosales, Sinaloa, México \\ Email: "parpolo@yahoo.com.mx
}

Received 17 February 2016; accepted 3 March 2016; published 7 March 2016

Copyright (C) 2016 by authors and OALib.

This work is licensed under the Creative Commons Attribution International License (CC BY). http://creativecommons.org/licenses/by/4.0/

(c) (i) Open Access

\section{Abstract}

To determine the efficacy of formulations $\mathrm{Ca}\left(\mathrm{NO}_{3}\right)_{2} \mathrm{liq}, \mathrm{Ca}\left(\mathrm{NO}_{3}\right)_{2}$ sol-1, $\mathrm{Ca}\left(\mathrm{NO}_{3}\right)_{2}$ sol-2 and $\mathrm{Ca}\left(\mathrm{NO}_{3}\right)_{2}$ sol-3, as well as the most appropriate dose of each formulation to induce the growth and yield of tomato (Lycopersicon esculentum Mill.), the research was conducted in greenhouses, to transplanting a plant per plastic pot with capacity of $12 \mathrm{~L}$, which were applied $21,20,19,18,17,16$ and

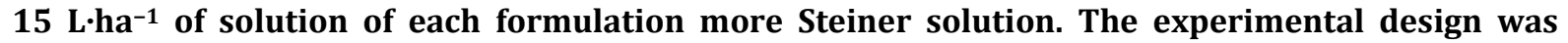
randomized complete block with three replicates per treatment. The best answer tomato was achieved with $\mathrm{Ca}\left(\mathrm{NO}_{3}\right)_{2}$ líq, followed by that seen with $\mathrm{Ca}\left(\mathrm{NO}_{3}\right)_{2}$ sol-1 source, but the best effect on fruit yield and dry weight of the same was observed with the dose of $15 \mathrm{~L}^{\cdot} \cdot \mathrm{ha}^{-1}$ of $\mathrm{Ca}\left(\mathrm{NO}_{3}\right)_{2}$ líq and $\mathrm{Ca}\left(\mathrm{NO}_{3}\right)_{2}$ sol-3, while $\mathrm{Ca}\left(\mathrm{NO}_{3}\right)_{2}$ sol-2 and $\mathrm{Ca}\left(\mathrm{NO}_{3}\right)_{2}$ sol-1 best were the 16 and $17 \mathrm{~L} \cdot \mathrm{ha}^{-1}$. In all cases of calcium sources, doses that better effect resulted in the growth and fruit yield were lower that the recommended commercially, indicating that for growing saladette tomato variety Rio Grande should use a dose lower that the recommended to meet the objectives of agricultural sustainability, for the conservation and improvement of natural resources (in this case the soil and tomato plants) and improving the environment.

\section{Keywords}

Variety “Rio Grande”, Yield, Sustainability, Environment, Natural Resources

Subject Areas: Plant Science

\footnotetext{
${ }^{*}$ Corresponding author.
}

How to cite this paper: Montenegro, H.M.M., Mares, V.Á., Ruvalcaba, L.P., Sarabia, J.A.M. and Terán, J.A.L. (2016) Response of the Saladette Tomato of Growth Determinate to Different Sources and Doses of Calcium in Greenhouse. Open Access Library Journal, 3: e2484. http://dx.doi.org/10.4236/oalib.1102484 


\section{Introducción}

El tomate (Lycopersicon esculentum Mill.) es la hortaliza más popular y aceptada en la cultura gastronómica del mundo, y se cultiva en más de cien países para consumo en fresco o para su industrialización. Los diez principales países productores que concentran más de 80\% del total mundial son: China, Estados Unidos, India, Egipto, Turquía, Italia, Irán, España, Brasil y México. Los tres primeros marcan las tendencias de precios y consumo mundiales, de acuerdo con la FAO [1].

Según Nieto y Velasco [2], en México se siembran anualmente alrededor de 80,000 ha, con un rendimiento promedio de $28.7 \mathrm{t} \cdot \mathrm{ha}^{-1}$ en condiciones de campo abierto, por lo cual es la hortaliza más importante en superficie sembrada, volumen en el mercado nacional, generación de empleos y valor de la producción. Además, González et al. [3] han reportado que en casi todos los países consumidores, la calidad del tomate fresco (sabor, color, tamaño, etc.) se sobrepone al precio y valor nutritivo al momento de la elección de compra por parte del consumidor final. Relacionado con esto, González y Martínez [4] también han referido que el tomate ha sido y es de gran importancia socioeconómica para México, de tal forma que según De Giglio [5], anualmente se exporta a Estados Unidos 90\% de la producción, principalmente en la temporada invernal.

De acuerdo con Lorenz y Maynard [6], los frutos de tomate se caracterizan por tener 94\% de agua, 19 kcal de energía, 0.9 g de proteínas, 0.2 g de grasa, 4.3 g de carbohidratos, 0.5 g de fibra, $7.0 \mathrm{mg}$ de Ca, $23 \mathrm{mg}$ de P, 0.5 $\mathrm{mg}$ de Fe, $8.0 \mathrm{mg}$ de $\mathrm{Na}, 207 \mathrm{mg}$ de K, $1133 \mathrm{IU}$ de vitamina A, $0.06 \mathrm{mg}$ de tiamina, $0.05 \mathrm{mg}$ de riboflavina, 0.60 $\mathrm{mg}$ de niacina, $17.6 \mathrm{mg}$ de ácido ascórbico y $0.05 \mathrm{mg}$ de vitamina $\mathrm{B}_{6}$.

El cultivo de tomate en condiciones protegidas incrementa el rendimiento y la calidad de fruto. De acuerdo con Fonseca [7] la superficie utilizada para cultivos en invernadero ascendió a 6000 ha y creció anualmente en un 25\%, de esta superficie, 3,450 ha se destinaron para la producción de tomate en México, aunque de acuerdo con la AMHPAC [8], para el año 2013 se utilizaron poco más de 12,000 ha para el cultivo de diversos cultivos en invernadero.

Los sistemas de producción varían en cuanto a variedades, sustratos de crecimiento, dosis de nutrimentos, técnicas de control de plagas y enfermedades, entre otros factores, de acuerdo con Rodríguez et al. [9].

Jaramillo et al. [10] mencionan que el sistema de producción de tomate (Lycopersicon esculentum Mill.) bajo condiciones protegidas en México es relativamente nuevo, generando un impacto importante en los últimos años, por su incremento, superficie cultivada, productividad, rentabilidad y calidad del producto. El rendimiento promedio obtenido con este sistema es entre 5 y $8 \mathrm{~kg}$ por planta, superando tres veces el que se obtiene a campo abierto, que está entre 1.5 y $2 \mathrm{~kg}$ por planta. SIACON [11] refieren que en México, la producción de tomate en 2008 fue de 2.3 millones de toneladas. Sin embargo, Cabrera [12], Howard [13] y Morel et al. [14] han reportado que el cultivo bajo invernadero requiere de ciertas condiciones y medios para llevarse a cabo. Uno de los principales factores que determinan el éxito es el sustrato o medio de crecimiento.

El calcio se absorbe en forma de ión $\mathrm{Ca}^{2+}$ divalente y los síntomas de su deficiencia siempre son más pronunciados en los tejidos jóvenes, así lo han mencionado Kirby y Pilbeam [15], de tal manera que las zonas meristemáticas de las raíces, tallos y hojas, donde existen divisiones celulares, son las más susceptibles, quizás porque se necesita calcio para que forme una nueva laminilla media en la placa celular que aparece entre las células hijas, según Salisbury y Ross [16].

Lea y Leegood [17] refieren que en la célula ocurren secuencias de reacciones que requiere $\mathrm{Mn}^{2+}, \mathrm{Ca}^{2+} \mathrm{y} \mathrm{Cl}^{-}$ ligados a un conjunto de polipéptidos, y otros datos experimentales sugieren que respuestas inducidas del fitocromo requieren intermediarios como el $\mathrm{Ca}^{2+}$ y calmodulina y que estas sustancias juegan un papel en la fosforilación de proteínas nucleares.

Sin embargo, Hepler y Wayne [18] señalan que actualmente se reconoce que todos los organismos mantienen concentraciones inesperadamente bajas de $\mathrm{Ca}^{2+}$ libre en el citosol, habitualmente menores de $1 \mu \mathrm{M}$, lo que de acuerdo con Kinzel [19] resulta cierto incluso cuando el calcio es tan abundante como el fósforo, azufre y magnesio, en muchas plantas, sobre todo leguminosas. No obstante, la mayor parte del calcio que contienen las plantas se encuentra en las vacuolas centrales, y en las paredes celulares se encuentra unido a ciertos polisacáridos llamados pectatos.

En las vacuolas, el calcio suele precipitarse en forma de cristales de oxalato insolubles. En algunas especies, también se encuentra en forma de carbonato, fosfato o sulfato insoluble. Las concentraciones bajas de calcio, casi micromolares, deben mantenerse en parte para impedir la formación de sales de calcio insolubles, obtenidas a partir de ATP y de otros fosfatos orgánicos. Además, las concentraciones de $\mathrm{Ca}^{2+}$ por encima del margen mi- 
cromolar inhiben la corriente citoplasmática, así lo ha mencionado Williamson [20].

El objetivo de esta investigación fue determinar la eficacia de las formulaciones de $\mathrm{Ca}\left(\mathrm{NO}_{3}\right)_{2}$ liq, $\mathrm{Ca}\left(\mathrm{NO}_{3}\right)_{2}$ sol-1, $\mathrm{Ca}\left(\mathrm{NO}_{3}\right)_{2}$ sol-2 y Ca( $\left(\mathrm{NO}_{3}\right)_{2}$ sol-3, así como la dosis más adecuada de cada formulación, para inducir el crecimiento y rendimiento de frutos del tomate saladette.

\section{Materiales y Métodos}

La presente investigación se realizó durante el ciclo agrícola otoño-invierno 2014-2015 en un invernadero tipo capilla, instalado en el campo experimental de la Universidad Tecnológica de Culiacán, ubicado en el km 2 de la carretera Culiacán-Imala, colonia Los Ángeles, Culiacán, Sinaloa, con coordenadas 2450'30"N y 10750'30"E, en altura de 58 msnm. Según García [21], el clima es [BS $\left.{ }_{1}\left(h^{\prime}\right) w(w)(e)\right]$ semiseco, muy cálido, extremoso con lluvias en verano, con una temperatura media anual de $25.9^{\circ} \mathrm{C}$ y una precipitación media anual de $672.8 \mathrm{~mm}$.

El cultivar de tomate saladette (Lycopersicon esculentum Mill.) utilizado fue "Río Grande" de crecimiento determinado, el cual se manejó en contenedores a base de bolsas de plástico con capacidad de $12 \mathrm{~L}$, tratadas por imbibición en solución con el hongo Trichoderma harzianum Rifai $\left(2 \times 10^{12} \mathrm{ufc} \cdot \mathrm{g}^{-1}\right)$ y las bacterias Bacillus subtilis (Ehrengerg) Cohn $\left(2 \times 10^{12} \mathrm{ufc} \cdot \mathrm{mL}^{-1}\right)$ y Bacillus thuringiensis Berliner $\left(2 \times 10^{12} \mathrm{ufc} \cdot \mathrm{mL}^{-1}\right)$, a fin de favorecer el crecimiento radicular y evitar el estrés post-trasplante. El trasplante se realizó el 19 de Octubre de 2014, con una planta por maceta. Las plantas se manejaron a un tallo tutorado con rafia de plástico sostenida en hilos de alambre tendidos horizontalmente a lo largo del invernadero; las podas se hicieron conforme las plantas lo requirieron. Las fuentes de calcio fueron $\mathrm{Ca}\left(\mathrm{NO}_{3}\right)_{2}$ liq con $10.5 \%$ de $\mathrm{N}$ total y $12.5 \%$ de $\mathrm{Ca}, \mathrm{Ca}\left(\mathrm{NO}_{3}\right)_{2}$ sol-1 con $10.1 \%$ de $\mathrm{N}$ total y $17.3 \%$ de $\mathrm{Ca}, \mathrm{Ca}\left(\mathrm{NO}_{3}\right)_{2}$ sol-2 con $15.5 \%$ de $\mathrm{N}$ total y $26.5 \%$ de $\mathrm{Ca}$ y $\mathrm{Ca}\left(\mathrm{NO}_{3}\right)_{2}$ sol-3 con $15.5 \%$ de $\mathrm{N}$ total y $26 \%$ de $\mathrm{Ca}$, a partir de las cuales se elaboraron las siete dosis o tratamientos siguientes: 21 , 20, 19, 18, 17, 16 y $15 \mathrm{~L} \cdot \mathrm{ha}^{-1}$, al considerar como base que son $18 \mathrm{~L} \cdot \mathrm{ha}^{-1}$ los que se recomiendan comercialmente (testigo uno) de cada fuente; de cada dosis se aplicaron $1200 \mathrm{~mL}$ por maceta tres veces por semana hasta el momento de la última cosecha de frutos; también se aplicó solución Steiner (testigo dos) diariamente, excepto cuando se regó con solución de calcio, durante todo el periodo del cultivo (hasta el tercer corte) con la misma cantidad de mL por maceta. El diseño experimental fue bloques completos al azar, con tres repeticiones (tres macetas con una planta cada una) por tratamiento.

Por medio de charolas colocadas por debajo de las macetas se capturó el agua drenada para analizar el pH y CE de la misma. Después de 18 días de haber aplicado los tratamientos, las muestras se tomaron cada tercer día, para hacer un total de 25 muestreos. $\mathrm{El} \mathrm{pH}=7.3$ a la entrada y $\mathrm{pH}=6.9$ de salida se determinó con un Peachimetro portátil, modelo HI-98127, marca HANNA, USA, y la CE $=0.75$ de entrada y CE $=1.3 \mathrm{dS} \cdot \mathrm{m}^{-1}$ también se determinó con conductivímetro portátil modelo HI-98331, marca HANNA, USA.

Las variables de estudio fueron clorofila, altura de plantas, diámetro del tallo, longitud y ancho de las hojas, peso fresco y seco de frutos, peso fresco y seco de plantas, así como el rendimiento por hectárea. El contenido de clorofila fue el número de unidades spad determinado con clorofilímetro portátil (Spad-502 marca Minolta, Japón), la altura se midió con el metro desde la superficie del sustrato hasta la parte más alta de la planta; asimismo, la longitud y ancho de las hojas, el diámetro del tallo se determinó con vernier digital, el peso seco se obtuvo mediante secado en estufa Red Line by Binder RI-115, Germany, a $85^{\circ} \mathrm{C}$ hasta peso constante, de tal forma que el peso fresco y seco de frutos y plantas, así como el rendimiento, se determinaron con báscula de precisión marca Ohaus, TJ-611, corporation USA.

Los datos se analizaron estadísticamente con el procedimiento proc glm del SAS Institute [22] versión 6.12, utilizando la prueba de Tukey con $\alpha \leq 0.05$ para la comparación múltiple de medias, en la mayoría de los casos, pero para las variables de peso fresco y seco de los frutos se aplicó la prueba de Duncan con $\alpha \leq 0.05$.

\section{Resultados Y Discusión}

Con un promedio de 51.7 unidades spad, un coeficiente de variación (CV) igual a 11.2\%, una desviación estándar (S) de 5.8 unidades spad y una probabilidad de cometer error (Pr) igual a 0.0171, se observaron diferencias estadísticas entre los promedios del contenido de clorofila (Cuadro 1), de tal manera que con la fuente de $\mathrm{Ca}\left(\mathrm{NO}_{3}\right)_{2}$ sol-1 se tuvo un incremento de $9.6 \%$ en comparación con el valor obtenido con la fuente de $\mathrm{Ca}\left(\mathrm{NO}_{3}\right)_{2}$ sol-2, mientras que con respecto a la media lograda con $\mathrm{Ca}\left(\mathrm{NO}_{3}\right)_{2}$ sol-3 el incremento fue de $6.1 \%$, en tanto que entre los promedios estimados con $\mathrm{Ca}\left(\mathrm{NO}_{3}\right)_{2}$ sol-1 y Ca( $\left(\mathrm{NO}_{3}\right)_{2}$ liq, la diferencia apenas fue de 0.4 unidades spad.

Entre los promedios de altura también ocurrieron diferencias estadísticas (Cuadro 1), con una media de la 
muestra de $69.5 \mathrm{~cm}$, un $\mathrm{CV}=11.3 \%$, una $\mathrm{S}=7.9 \mathrm{~cm}$ y una $\mathrm{Pr}=0.0001$, pero las plantas que más crecieron en este aspecto fueron aquellas cultivadas con las fuentes de $\mathrm{Ca}\left(\mathrm{NO}_{3}\right)_{2}$ sol-2 y Ca( $\left(\mathrm{NO}_{3}\right)_{2}$ sol-3, con la primer fuente los incrementos fueron de 10.5 y $14.9 \%$ comparados con los promedios logrados con $\mathrm{Ca}\left(\mathrm{NO}_{3}\right)_{2}$ liq y $\mathrm{Ca}\left(\mathrm{NO}_{3}\right)_{2}$ sol-1, pero con la segunda fuente los incrementos fueron de 12.4 y $16.7 \%$ con respecto a los valores estimados en las plantas cultivadas con $\mathrm{Ca}\left(\mathrm{NO}_{3}\right)_{2}$ liq y $\mathrm{Ca}\left(\mathrm{NO}_{3}\right)_{2}$ sol-1, mientras que entre los promedios logrados con $\mathrm{Ca}\left(\mathrm{NO}_{3}\right)_{2}$ liq y $\mathrm{Ca}\left(\mathrm{NO}_{3}\right)_{2}$ sol-1, la diferencia sólo fue de $2.5 \mathrm{~cm}$, lo que significó un ligero incremento de $3.9 \%$ de las plantas manejadas con $\mathrm{Ca}\left(\mathrm{NO}_{3}\right)_{2}$ liq. No obstante, la correlación de clorofila con la altura de las plantas tuvo un porcentaje de $86 \%$, pero no fue significativa $(\operatorname{Pr}=0.1317)$.

Para el diámetro del tallo se obtuvo una media de la muestra de $10.0 \mathrm{~mm}, \mathrm{CV}=11.1 \%, \mathrm{~S}=1.1 \mathrm{~mm}$ y $\operatorname{Pr}=$ 0.5010; sin embargo, los promedios obtenidos con cada una de las fuentes de calcio fueron muy similares (Cuadro 1), pero la correlación de clorofila con el diámetro del tallo alcanzó un porcentaje de 49\%, pero tampoco fue significativa $(\mathrm{Pr}=0.5089)$.

Las diferencias de crecimiento en altura y diámetro del tallo indican que en las zonas meristemáticas de las raíces y tallos, las divisiones celulares ocurrieron de manera más limitada con algunas fuentes de $\mathrm{Ca}^{2+}$ que con otras y, en consecuencia, como lo señalan Salisbury y Ross [16], con unas fuentes fue más lenta la formación de las nuevas laminillas medias en la placa celular que aparece entre las células hijas, en las cuales también, de acuerdo con Lea y Leegood [17], se requirió para la fosforilación de proteínas nucleares, pero con las diferencias inducidas por las fuentes de $\mathrm{Ca}^{2+}$.

Con respecto a la longitud y ancho de las hojas, así como al peso fresco y seco de las plantas, no se observaron diferencias estadísticas (Cuadro 2), pero la media de la muestra en longitud de hojas fue de $28.1 \mathrm{~cm}$, el CV $=17.2 \%$, la $\mathrm{S}=4.8 \mathrm{~cm}$ y la $\mathrm{Pr}=0.9055$; para lo ancho de las hojas los respectivos valores fueron $28.1 \mathrm{~cm}$, 20.1\%, 5.7 cm y 0.5790; en el peso fresco de las plantas los valores fueron de 188.4 g, 39.9\%, $75.3 \mathrm{~g}$ y 0.2341 ; y para el peso seco fueron de $47.8 \mathrm{~g}, 34.2 \%, 16.3 \mathrm{~g}$ y 0.8322 .

Sin embargo, específicamente en el peso seco, las plantas cultivadas con calcio líquido acumularon $4.0 \%$ más de materia seca en comparación con lo acumulado por aquéllas que fueron manejadas con $\mathrm{Ca}\left(\mathrm{NO}_{3}\right)_{2}$ sol-2,

Cuadro 1. Contenido de clorofila, altura y diámetro del tallo de plantas de tomate saladette cultivado con cuatro formulaciones de calcio.

\begin{tabular}{|c|c|c|c|}
\hline Formulaciones de calcio & Contenido de clorofila (unid. spad) & Altura de plantas (cm) & Diámetro del tallo (mm) \\
\hline $\mathrm{Ca}\left(\mathrm{NO}_{3}\right)_{2}$ liq & $53.3 \mathrm{ab}$ & $66.3 \mathrm{~b}$ & $10.3 \mathrm{a}$ \\
\hline $\mathrm{Ca}\left(\mathrm{NO}_{3}\right)_{2}$ sol-1 & $53.7 \mathrm{a}$ & $63.8 \mathrm{~b}$ & $10.0 \mathrm{a}$ \\
\hline $\mathrm{Ca}\left(\mathrm{NO}_{3}\right)_{2}$ sol-2 & $49.0 \mathrm{~b}$ & $73.3 \mathrm{a}$ & $10.0 \mathrm{a}$ \\
\hline $\mathrm{Ca}\left(\mathrm{NO}_{3}\right)_{2}$ sol-3 & $50.6 \mathrm{ab}$ & 74.5 a & $10.0 \mathrm{a}$ \\
\hline DMSH & 4.3 & 3.7 & 0.8 \\
\hline
\end{tabular}

Medias con la misma letra en cada columna son estadísticamente iguales (Tukey, $\alpha \leq 0.05$ ).

Cuadro 2. Promedios de longitud y anchura de hojas, peso fresco y seco de plantas de tomate saladette cultivado con cuatro formulaciones de calcio.

\begin{tabular}{|c|c|c|c|c|c|}
\hline $\begin{array}{c}\text { Formula } \\
\text { ciones de calcio }\end{array}$ & $\begin{array}{l}\text { Longitud de } \\
\text { hojas }(\mathrm{cm})\end{array}$ & $\begin{array}{l}\text { Ancho de hojas } \\
\qquad(\mathrm{cm})\end{array}$ & $\begin{array}{l}\text { Peso fresco de plan- } \\
\text { tas (g) }\end{array}$ & $\begin{array}{l}\text { Peso seco de } \\
\text { plantas (g) }\end{array}$ & $\begin{array}{l}\text { Rendimiento de } \\
\text { frutos } / \mathrm{m}^{2} \text { (g) }\end{array}$ \\
\hline $\mathrm{Ca}\left(\mathrm{NO}_{3}\right)_{2}$ liq & $27.8 \mathrm{a}$ & $27.3 \mathrm{a}$ & $197.3 \mathrm{a}$ & $49.2 \mathrm{a}$ & 586.9 a \\
\hline $\mathrm{Ca}\left(\mathrm{NO}_{3}\right)_{2}$ sol-1 & 28.5 a & $29.0 \mathrm{a}$ & $194.6 \mathrm{a}$ & $49.1 \mathrm{a}$ & $501.0 \mathrm{ab}$ \\
\hline $\mathrm{Ca}\left(\mathrm{NO}_{3}\right)_{2}$ sol-2 & 28.5 a & $29.0 \mathrm{a}$ & $161.0 \mathrm{a}$ & $47.3 \mathrm{a}$ & $392.8 \mathrm{~b}$ \\
\hline $\mathrm{Ca}\left(\mathrm{NO}_{3}\right)_{2}$ sol-3 & $27.8 \mathrm{a}$ & $27.3 \mathrm{a}$ & 200.9 a & $45.4 \mathrm{a}$ & $390.2 \mathrm{~b}$ \\
\hline DMSH & 3.6 & 4.3 & 56.9 & 12.4 & 213.9 \\
\hline
\end{tabular}

Medias con la misma letra en cada columna son estadísticamente iguales (Tukey, $\alpha \leq 0.05$ ). 
pero con respecto a las cultivadas con $\mathrm{Ca}\left(\mathrm{NO}_{3}\right)_{2}$ sol-3, el incremento fue de $8.4 \%$. Las plantas que recibieron $\mathrm{Ca}\left(\mathrm{NO}_{3}\right)_{2}$ sol-1 también acumularon más materia seca que aquéllas manejadas con $\mathrm{Ca}\left(\mathrm{NO}_{3}\right)_{2}$ sol-2 y $\mathrm{Ca}\left(\mathrm{NO}_{3}\right)_{2}$ sol-3, y los respectivos incrementos fueron de 3.8 y $8.1 \%$. No obstante, entre las cuatro variables y el contenido de clorofila la correlación no fue significativa.

El análisis de varianza arrojó una media de la muestra de $467.7 \mathrm{~g} \cdot \mathrm{m}^{-2}$ de rendimiento, con $\mathrm{CV}=30.3 \%, \mathrm{~S}=$ 141.6 g y $\operatorname{Pr}=0.0513$, y este carácter se expresó con las diferencias estadísticas que se indican en el Cuadro 2, en donde se puede observar que con el nitrato de calcio líquido esta variable se incrementó en 49.4 y $50.4 \%$ con respecto al rendimiento que se obtuvo con las soluciones de $\mathrm{Ca}\left(\mathrm{NO}_{3}\right)_{2}$ sol-2, $\mathrm{Ca}\left(\mathrm{NO}_{3}\right)_{2}$ sol-2 y Ca( $\left(\mathrm{NO}_{3}\right)_{2}$ sol-3, mientras que con $\mathrm{Ca}\left(\mathrm{NO}_{3}\right)_{2}$ sol-1, los incrementos fueron de 27.5 y $28.4 \%$ en comparación a los promedios logrados con las mismas soluciones $\mathrm{Ca}\left(\mathrm{NO}_{3}\right)_{2}$ sol-2 y Ca( $\left(\mathrm{NO}_{3}\right)_{2}$ sol-3). El incremento del peso seco de las plantas y del rendimiento de frutos $/ \mathrm{m}^{2}$ quizás se debió a lo que menciona Millar et al. [23], ya que éstos refieren que ante una saturación de bases menor al 100\%, el aumento del pH está asociado con el incremento en la cantidad de $\mathrm{Ca}^{2+}$ en la solución del suelo, puesto que el $\mathrm{Ca}^{2+}$ es una de las bases usualmente intercambiables dominantes; además, muchos estudios relacionan el aumento en el crecimiento de las plantas con el incremento en el porcentaje de $\mathrm{Ca}^{2+}$ en las plantas y con el mayor valor del $\mathrm{pH}$ o porcentaje de saturación de bases.

Con la dosis de $15 \mathrm{~L} \cdot h a^{-1}$ de $\mathrm{Ca}\left(\mathrm{NO}_{3}\right)_{2}$ liq, el promedio de peso fresco de frutos superó en $42.9 \%$ al promedio que se obtuvo con la solución Steiner y en $165.2 \%$ al que se logró con la dosis comercial $\left(18 \mathrm{~L} \cdot \mathrm{ha}^{-1}\right)$ de dicha formulación (Cuadro 3); con la dosis de $15 \mathrm{~L} \cdot \mathrm{ha}^{-1}$ de $\mathrm{Ca}\left(\mathrm{NO}_{3}\right)_{2}$ sol-1 el promedio de peso fresco fue igual al que se obtuvo con la solución Steiner, pero superó en $175.4 \%$ al que se logró con la dosis comercial, mientras que con las dosis de 16, 17, 19, 20 y $21 \mathrm{~L} \cdot \mathrm{ha}^{-1}$ los incrementos fueron de 167.8, 57.8, 75.3, 55.3 y 156.4\%, respectivamente.

Con el Ca( $\left(\mathrm{NO}_{3}\right)_{2}$ sol-2 la mejor dosis también fue la de $15 \mathrm{~L} \cdot h a^{-1}$, ya que el peso fresco superó en los respectivos 96.5 y $44.6 \%$ a lo que se obtuvo con la solución Steiner y la dosis comercial, incluso superó en 28.2, 13.6, 62.2, 62.2 y 134.9\% a lo que se logró con las dosis de 16, 17, 19, 20 y $21 \mathrm{~L}^{2} \mathrm{ha}^{-1}$, respectivamente. Con $\mathrm{Ca}\left(\mathrm{NO}_{3}\right)_{2}$ sol-3 la mejor dosis también fue la de $15 \mathrm{~L} \cdot h a^{-1}$, ya que con élla el incremento fue de 13.9 y $21.6 \%$ en comparación a los promedios obtenidos con solución Steiner y la dosis comercial, pero con la misma dosis también se lograron incrementos de 63.4, 15.1, 65.4, 145.1 y 36.5\% con relación a los promedios logrados con las dosis de 16, 17, 19, 20 y $21 \mathrm{~L} \cdot \mathrm{ha}^{-1}$, respectivamente.

En el peso seco de los frutos no se observaron diferencias estadísticas significativas, pero puede notarse que con la dosis de $15 \mathrm{~L} \cdot \mathrm{ha}^{-1}$ de $\mathrm{Ca}\left(\mathrm{NO}_{3}\right)_{2}$ liq el peso se incrementó en 16.1 y $13.2 \%$ comparado con lo logrado a través de la solución Steiner y la dosis comercial, respectivamente (Cuadro 4). Además, también superó en 97.5, 45.1, 60.9, 116.4 y $73.2 \%$ al peso seco de los frutos cosechados de las plantas cultivadas con las respectivas dosis de $16,17,19,20$ y $21 \mathrm{~L} \cdot \mathrm{ha}^{-1}$.

Cuadro 3. Peso fresco de frutos de tomate saladette cultivado con diferentes fuentes y dosis de calcio.

\begin{tabular}{|c|c|c|c|c|}
\hline Dosis/fuentes & $\mathrm{Ca}\left(\mathrm{NO}_{3}\right)_{2}$ liq & $\mathrm{Ca}\left(\mathrm{NO}_{3}\right)_{2}$ sol-1 & $\mathrm{Ca}\left(\mathrm{NO}_{3}\right)_{2}$ sol-2 & $\mathrm{Ca}\left(\mathrm{NO}_{3}\right)_{2}$ sol-3 \\
\hline de calcio & \multicolumn{4}{|c|}{ Peso fresco de frutos (g) } \\
\hline S. Steiner & $138.3 \mathrm{ab}$ & $181.4 \mathrm{a}$ & $118.7 \mathrm{~b}$ & 238.2 a \\
\hline $21 \mathrm{~L} \cdot \mathrm{ha}^{-1}$ & $190.5 \mathrm{ab}$ & $168.2 \mathrm{a}$ & $99.3 \mathrm{~b}$ & 198.7 a \\
\hline $20 \mathrm{~L} \cdot \mathrm{ha}^{-1}$ & $184.2 \mathrm{ab}$ & 101.9 a & $143.8 \mathrm{ab}$ & $110.7 \mathrm{a}$ \\
\hline $19 \mathrm{~L} \cdot \mathrm{ha}^{-1}$ & $129.0 \mathrm{ab}$ & 115.0 a & $143.8 \mathrm{ab}$ & $164.0 \mathrm{a}$ \\
\hline $18 \mathrm{~L} \cdot \mathrm{ha}^{-1}$ & $74.5 \mathrm{ab}$ & $65.6 \mathrm{a}$ & $161.3 \mathrm{ab}$ & 223.0 a \\
\hline $17 \mathrm{~L} \cdot \mathrm{ha}^{-1}$ & $53.5 \mathrm{~b}$ & $103.5 \mathrm{a}$ & $205.3 \mathrm{ab}$ & 235.7 a \\
\hline $16 \mathrm{~L} \cdot \mathrm{ha}^{-1}$ & $87.2 \mathrm{ab}$ & 175.7 a & $182.0 \mathrm{ab}$ & $166.0 \mathrm{a}$ \\
\hline $15 \mathrm{~L} \cdot \mathrm{ha}^{-1}$ & 197.6 a & 180.7 a & 233.3 a & 271.3 a \\
\hline DMSH & 141.1 & 172.5 & 108.4 & 251.5 \\
\hline
\end{tabular}

Medias con la misma letra en cada columna son estadísticamente iguales (Duncan, $\alpha \leq 0.05$ ). 
Cuadro 4. Peso seco de frutos de tomate saladette cultivado con diferentes fuentes y dosis de calcio.

\begin{tabular}{|c|c|c|c|c|}
\hline Dosis/fuentes & $\mathrm{Ca}\left(\mathrm{NO}_{3}\right)_{2}$ liq & $\mathrm{Ca}\left(\mathrm{NO}_{3}\right)_{2}$ sol-1 & $\mathrm{Ca}\left(\mathrm{NO}_{3}\right)_{2}$ sol-2 & $\mathrm{Ca}\left(\mathrm{NO}_{3}\right)_{2}$ sol-3 \\
\hline de calcio & \multicolumn{4}{|c|}{ Peso seco de frutos (g) } \\
\hline S. Steiner & $54.6 \mathrm{a}$ & $23.7 \mathrm{a}$ & $41.8 \mathrm{a}$ & $47.4 \mathrm{a}$ \\
\hline $21 \mathrm{~L} \cdot \mathrm{ha}^{-1}$ & $36.6 \mathrm{a}$ & $18.1 \mathrm{a}$ & $53.3 \mathrm{a}$ & $58.2 \mathrm{a}$ \\
\hline $20 \mathrm{~L} \cdot \mathrm{ha}^{-1}$ & $29.3 \mathrm{a}$ & $18.3 \mathrm{a}$ & $46.4 \mathrm{a}$ & 36.9 a \\
\hline $19 \mathrm{~L} \cdot \mathrm{ha}^{-1}$ & $39.4 \mathrm{a}$ & $23.3 \mathrm{a}$ & $50.8 \mathrm{a}$ & $32.4 \mathrm{a}$ \\
\hline $18 \mathrm{~L} \cdot \mathrm{ha}^{-1}$ & $56.0 \mathrm{a}$ & $16.4 \mathrm{a}$ & $43.2 \mathrm{a}$ & $46.2 \mathrm{a}$ \\
\hline $17 \mathrm{~L} \cdot \mathrm{ha}^{-1}$ & $43.7 \mathrm{a}$ & $21.3 \mathrm{a}$ & $56.0 \mathrm{a}$ & $43.2 \mathrm{a}$ \\
\hline $16 \mathrm{~L} \cdot \mathrm{ha}^{-1}$ & $32.1 \mathrm{a}$ & $24.1 \mathrm{a}$ & 36.6 a & $41.4 \mathrm{a}$ \\
\hline $15 \mathrm{~L} \cdot \mathrm{ha}^{-1}$ & $63.4 \mathrm{a}$ & $18.0 \mathrm{a}$ & $50.2 \mathrm{a}$ & $57.7 \mathrm{a}$ \\
\hline DMSH & 70.6 & 13.3 & 36.4 & 60.7 \\
\hline
\end{tabular}

Medias con la misma letra en cada columna son estadísticamente iguales (Duncan, $\alpha \leq 0.05$ ).

Con el $\mathrm{Ca}\left(\mathrm{NO}_{3}\right)_{2}$ sol-1 tampoco se observaron diferencias estadísticas significativas, pero el mayor promedio de peso seco se logró con la dosis de $16 \mathrm{~L} \cdot \mathrm{ha}^{-1}$, el cual superó en 1.7 y $46.9 \%$ al peso obtenido con solución Steiner y la dosis comercial; además, también superó en 33.9, 13.1, 3.4, 31.7 y 33.1\% a los promedios logrados con las respectivas dosis de 15, 17, 19, 20 y $21 \mathrm{~L} \cdot \mathrm{ha}^{-1}$. Con el $\mathrm{Ca}\left(\mathrm{NO}_{3}\right)_{2}$ sol-2, la mejor respuesta se observó donde se aplicó la dosis de $17 \mathrm{~L} \cdot \mathrm{ha}^{-1}$, ya que con dicha dosis el promedio de peso seco de los frutos cosechados se incrementó en 34.0\% en relación al promedio obtenido con la solución Steiner y en 29.6\% comparado con el promedio estimado en las plantas cultivadas con la dosis comercial; con respecto a los promedios logrados con las dosis de 15, 16, 19, 20 y $21 \mathrm{~L} \cdot \mathrm{ha}^{-1}$, los incrementos fueron de 11.5, 53.0, 10.2, 20.7 y 5.1\%, respectivamente; mientras que con el $\mathrm{Ca}\left(\mathrm{NO}_{3}\right)_{2}$ sol-3 la mejor dosis fue la de $15 \mathrm{~L} \cdot \mathrm{ha}^{-1}$, ya que el promedio de peso seco de los frutos se incrementó en $21.7 \%$ con respecto a lo logrado con la solución Steiner y en $24.9 \%$ comparado con el promedio obtenido con la dosis comercial. Aunque también ocasionó incrementos de 39.4, 33.6, 78.1 y 56.4 con relación a los promedios estimados del mismo peso de los frutos cosechados de las plantas manejadas con las dosis de 16, 17, 19, 20 y $21 \mathrm{~L} \cdot h a^{-1}$.

De acuerdo con Millar et al. [23], el incremento del peso seco de los frutos, con cualquiera de las fuentes de $\mathrm{Ca}^{2+}$ utilizadas en este experimento, denota que este elemento fue absorbido por las plantas de tomate, para formar parte de la pared celular y repercutir en el crecimiento de las plantas y de los frutos. Estos resultados también tienen relación con lo que reportan Salisbury y Ross [15], ya que al existir más calcio disponible éste puede ser absorbido e incorporado en las partes celulares y, en consecuencia, influir en la división de las células para inducir el crecimiento y peso de los frutos que son dos componentes del rendimiento de tomate por unidad de superficie. Es decir, dependiendo del estado del ión calcio y la dosis, en este caso cada una de las formulaciones de donde se elaboraron las dosis, será la facilidad con que éste pueda ser absorbido por las plantas.

\section{Conclusiones}

La mejor respuesta de las plantas de tomate saladette se logró con la formulación de $\mathrm{Ca}\left(\mathrm{NO}_{3}\right)_{2}$ liq, seguida de la que se observó con la formulación de $\mathrm{Ca}\left(\mathrm{NO}_{3}\right)_{2}$ sol-1, pero en cuanto al efecto deseado en el rendimiento de frutos y en peso seco de los mismos mediante las dosis, las que mayor efecto ocasionaron fueron las de 15 $\mathrm{L} \cdot \mathrm{ha}^{-1}$ de $\mathrm{Ca}\left(\mathrm{NO}_{3}\right)_{2}$ liq y $\mathrm{Ca}\left(\mathrm{NO}_{3}\right)_{2}$ sol-3, en tanto que con $\mathrm{Ca}\left(\mathrm{NO}_{3}\right)_{2}$ sol-1 y Ca( $\left(\mathrm{NO}_{3}\right)_{2}$ sol-2 las mejores fueron

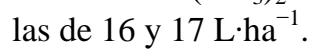

En todos los casos de fuentes de calcio, las dosis que mejor efecto ocasionaron en el crecimiento y rendimiento de frutos fueron inferiores a la recomendada comercialmente, lo que indica que al cultivar tomate saladette de la variedad Río Grande se debe utilizar una dosis más baja a la recomendada, para cumplir con los objetivos de la sustentabilidad agrícola que consisten en la conservación y mejoramiento de los recursos naturales (en este caso el suelo y las plantas de tomate) y en la mejora del medio ambiente. 


\section{References}

[1] FAO, Food Agricultural Organization (2012) Organización de las Naciones Unidas para la Agricultura y la Alimentación. www.apps.fao.org

[2] Nieto, A.R. and Velasco, H.E. (2006) Cultivo de Jitomate en Hidroponia e Invernadero. 2nd Edition, Departamento de Fitotecnia, Universidad Autónoma Chapingo. Chapingo, México, 130 p.

[3] González, C.A., Salas, S.M. del C. and Urrestarazu, G.M. (2004) Producción y calidad en el cultivo de tomate cherry. In: Urrestarazu Gavilán, M., Ed., Tratado de Cultivos sin Suelo, Mundi-Prensa. Madrid, España, 703-747.

[4] González, S.R.F. and Martínez, D.M.A. (2002) Dumping: El caso del jitomate, México 1996. Comunicaciones en Socioeconomía Estadística e Informática, 6, 1-30.

[5] DeGiglio, M.A. (2003) Growth of the Fresh Greenhouse Tomato Market in the USA. Acta Horticulturae, 611, 91-92. http://dx.doi.org/10.17660/ActaHortic.2003.611.15

[6] Lorenz, O.A. and Maynard, D.N. (1988) Knott’s Handbook for Vegetable Growers. 3rd Edition, Jhon Wiley \& Sons, Inc., Hoboken, 456 p.

[7] Fonseca, A.E. (2006) Producción de tomate en invernadero. In: Olivares, E.S., Ed., Cuarto Simposio Internacional de Producción de Cultivos en Invernadero, UANL, Facultad de Agronomía, Monterrey, N.L., 1-8.

[8] AMHPAC (2013) Agroprotección, presente y futuro agrícola. www.amhpac.org/portal/index.php?option=com_content\&view=article\&id=1680:agroproteccion-presente-y-futuro-agr icola\&catid=13:noticias\&Itemid=10

[9] Rodríguez, D.N., Cano, R.P., Figueroa, V.U., Palomo, G.A., Favela, Ch.E., Álvarez, R.V.P., Márquez, H.C. and Moreno, R.A. (2008) Producción de tomate en invernadero con humus de lombriz como sustrato. Revista Fitotecnia Mexicana, 31, 265-272.

[10] Jaramillo, J., Rodríguez, V. P., Guzmán, M. and Zapata, M. (2006) El cultivo de tomate bajo invernadero. Corpoica, Centro de Investigación La Selva, Rionegro (Antioquia, Colombia), 48 p.

[11] SIACON (2015) Sistema de Información Agropecuaria de Consultado en. www.siea.sagarpa.gob.mx/sistemas/siacon/SIACON.html

[12] Cabrera, R. (1999) Propiedades, uso y manejo de sustratos de cultivo para la producción de plantas en maceta. Revista Chapingo Serie Horticultura, 5, 5-11.

[13] Howard, M. (1998) Hydroponic Food Production. EUA, Santa Bárbara, CA, 520 p.

[14] Morel, P., Poncet, L. and Riviére, L. (2000) Les supports de cultura horticoles. Les Matériaux Complémentaries Ternatifs a la tourbe. INRA, Paris, $87 \mathrm{p}$.

[15] Kirkby, E.A. and Pilbeam, D.J. (1984) Calcium as a Plant Nutrient. Plant, Cell and Environment, 7, 397-405. http://dx.doi.org/10.1111/j.1365-3040.1984.tb01429.x

[16] Salisbury, F.B. and Ross, C.W. (2000) Fisiología de las Plantas. Paraninfo Thomson Learning, Madrid, 988 p.

[17] Lea, J.P. and Leegood, C.R. (1993) Photosynthetic Formation of ATP and NADPH and Regulatory Mechanisms. In: Buchanan, B.B., Ed., Plant Biochemistry and Molecular Biology, John Wiley \& Sons, Chichester, 10-231.

[18] Hepler, P.K. and Wayne, R.O. (1985) Calcium and Plant Development. Annual Review of Plant Physiology, 36, 397439. http://dx.doi.org/10.1146/annurev.pp.36.060185.002145

[19] Kinzel, H. (1989) Calcium in the Vacuoles and Cell Walls of Plant Tissue. Flora, 182, 99-125.

[20] Williamson, R.E. (1984) Calcium and the Plant Cytoskeleton. Plant, Cell and Environment, 3, 431-440. http://dx.doi.org/10.1111/j.1365-3040.1984.tb01433.x

[21] García, A.E. (2004) Modificaciones al Sistema de Clasificación Climática de Köppen. Instituto de Geografía-UNAM, México, 93 p.

[22] SAS Institute (1985) SAS User’s Guide: Basics. 5th Edition, SAS Institute Inc., Cary, 1181-1191.

[23] Millar, C.E., Turk, L.M. and Foth, H.D. (1980) Fundamentos de la Ciencia del Suelo. Compañía Editorial Continental, S. A. México, D.F., 527 p. 